description of this Table, it seems most probable that this substance is distinct from Factor-E. The former may be either a diasteroisomer or a polymer of the latter. Consequently, Factor-E has been proved to be the new diketopiperazine having the above mentioned structure.

Acknowledgements. The author wishes to express his sincere thanks to Prof. K. Sakaguchi, Univ. of Tokyo for his constant guidance throughout this work, and also to Prof. Y. Sumiki, Prof. T. Asai and Prof. M. Matsui, Univ. of Tokyo for their kind advice and suggestions extended throughout this work. Thanks are also due to Prof. S. Akabori, Univ. of Osaka for his generous supply of DL-proline, and to Mr. I. Matsumoto, Banyu Pharmaceutical Co. Ltd. for his kind supply of phosgene, further to Mr. K, Sugimura, Food Research Institute and Mr. J. Kirimura, Sericultural Experiment Station, Ministry of Agriculture and Forestry, for their help in the microbioassay. The micro analysis and infrared spectrum were carried out at this department.

[Bull. Agr. Chem. Soc. Japan, Vol. 22, No. 2, p. 103 108, 1958]

\title{
Biochemical Studies of Piricularia oryzae
}

\author{
Part V. Behavior towards DL-Norleucine and DL-Alanine.* \\ By Tokuji Shimomura and Yukihiko Nakamura \\ Department of Agricultural Chemistry, Faculty of Agriculture, Hokkaido University
}

Received November 13, 1957

\begin{abstract}
In the current experiment, the conversion of DL-norleucine and DL-alanine to their respective $\alpha$-keto analogue by Piricularia oryzae was confirmed by the separation of 2,4-dinitrophenylhydrazone of either keto acid. Although the formation of keto acid from norleucine was distinctly recognized both in the presence and the absence of arsenite, the accumulation of pyruvic acid from alanine was only found in the presence of arsenite, as it has been observed with some microorganisms. In addition, the preferential utilization of alanine to a proper carbon source was identified, which may provide a step towards the investigation of the nutritional relationship between the blast fungus and the host plant.
\end{abstract}

It is apparent that the study of the biochemical behavior of Piricularia oryzae towards nitrogenous compounds, especially amino acids, is of fundamental importance in order to prevent the host plant from the blast disease. From such a point of view, the utilization process of several amino acids by this fungus has been investigated by the alithor $s^{1,2,3,4,5)}$ and the conversion of the amino

* Presented in part at the Annual Meeting of the Agricultural Chemical Society of Japan, Tokyo, April 1, 1956.

1) Y. Nakamura and T. Shimomura, J. Agr. Chem. Soc. Japan, 27, 694, (1953).

2) Y. Nakamura, T. Shimomura and T. Sugimoto, Mertoirs, Fac. Aar., Hakkaido Univ., 2, 30, (1955).

3) T. Shimomura and Y. Nakamura, This Bulletin, 19, 178. (1955).

(9) Y. Nakamura and T. Shimomura. the Annual Meeting of Agr. Chem. Soc., Tokyo, April 1, (1955).

5) T. Shimomura and Y. Nakamura, part VI of this series. 
acid to respective $\alpha$-keto analogue was recognized. In current experiments, DL-norleucihe and DL-alanine were taken as the amino acid to be tested. As already reported, the six carbon amino acids such as leucine1) and isoleucine ${ }^{5)}$ have been examined in regard of their conversion to respective $\alpha$-keto acid and thus the remaining six carbon amino acid, that is, norleucine was naturally taken into question as the supplement, although its natural occurrence is still questionable at present ${ }^{6}$, in spite of the earlier works of Abderhalden et al.. On the other hand; the general metabolic significance of alanine has been well known and it was reported by Tomizawa7) and $O \operatorname{tani}{ }^{8}$ ) that this amino acid is effective as a nitrogen source in the same extent as asparagine, aspartic acid, glutamic acid, and glycine, but metabolic conversion of these amino acids was unstated. Tanaka's, reported that glutamic acid could be utilized preferentially to a proper carbon source by this fungus and proceeded that this fact might be interpreted as one of the biochemical mechanisms involved in the invasion of blast fungus in the host plant and its further growth. Similar results have been obtained with alanine by the authors.

The present communication records the utilization process of both amino acids just mentioned, the separation and identification of either keto acid and the preferential utilization of alanine to a proper carbon source, D-glucose.

\section{EXPERIMENTAL AND RESULTS}

Incubation--Two mycelial felts were incubated at $27-8^{\circ}$ in $60 \mathrm{ml}$ of complete medium $\mathrm{m}^{3)}$ containing $100 \mathrm{mg}$ of DL-norleucine or DL-alanine in place of sucrose. In the inhibition experiment, $5 \mathrm{ml}$ of $\mathrm{M} / 20$ potassium arsenite was substituted for $5 \mathrm{ml}$ of water. Ammoniaand amino-nitrogen in the culture filtrate during the

6) R. Consden, A. H. Gordon, A. J.P. Martin, O. Rosenheim and R. L. M. Synge, Biacbem. J., 39, 251, (1945).

7) T. Tomizawa, Ann. Phylopatbolog. Soc. Japan, 17, 113 , (1953).

8) Y. Otani, Ann. Phytopatholog. Sor. Japan, 17, 9, (1952).

9) S. Tanaka, Ann. Rep. Co-operai. Research (Agr.), Minist. Educat., 1956, 92.

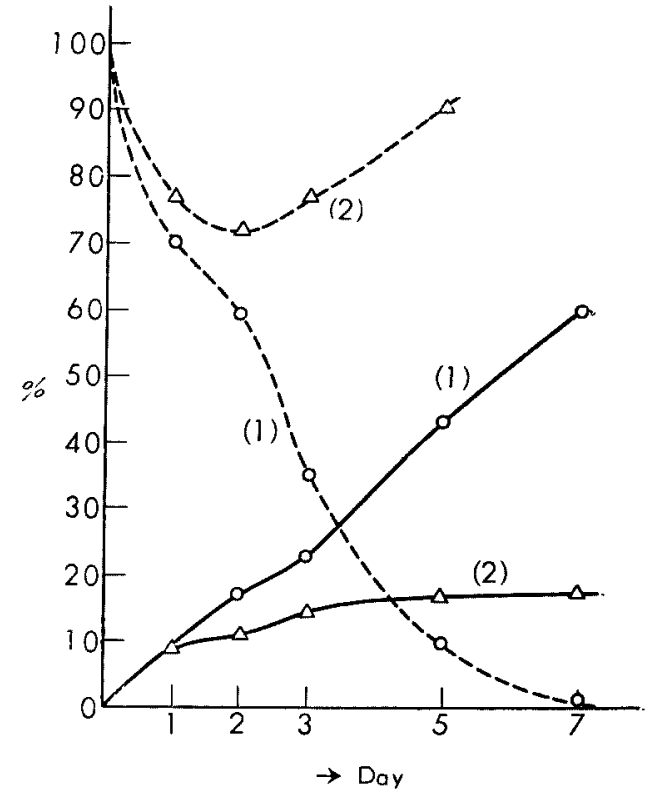

FIG. 1. Ammonia- and Amino-Nitrogen from DLNorleucine by $P$. oryzae.

(1). DL-norleucine only; (2), DL-norleucine + arsenite, Full line expresses ammonia-nitrogen and dotted, aminonitrogen. Values are indicated as percentage to theoretical amount of nitrogen of norleucine added as supplement. Incubation condition, see text.

incubation were determined as previously reported (Fig. 1 and Fig. 2). The same determinations were made with the culture medium consisting of $30 \mathrm{ml}$ of $\mathrm{M} / 15$ phosphate buffer ( $\mathrm{pH} 6.9$ ), $100 \mathrm{mg}$ of DL-alanine and $30 \mathrm{ml}$ of water and similar results as in the complete medium just mentioned were obtained (Fig. 3). This fact indicates that it is possible to carry out such experiment in a phosphate buffer medium without the addition of other inorganic salt. The determination of pyruvic acid accumulated in the phosphate buffer medium was made by a modification of the FriedemanHaugen method by Shimizu ${ }^{10)}$ (Fig. 4).

In order to ascertain whether $P$. oryzae is able to. utilize preferentially alanine to normal carbon source, this fungus was cultured at $27-8^{\circ}$ in $30 \mathrm{ml}$ of the medium containing $150 \mathrm{ml}$ of DL-alanine, $792.5 \mathrm{mg}$ of D-glucose and the same amount of inorganic salts and growth-factors as in the complete medium. The amount of carbon involved in present medium corresponded to that of $900 \mathrm{mg}$ of sucrose which had been. used as carbon source in normal cultivation. It was.

10) F. Egami et al., Standard Methods of Biochem. Experiments, . Bunkodo, Tokyo, (1953), P. 36. 


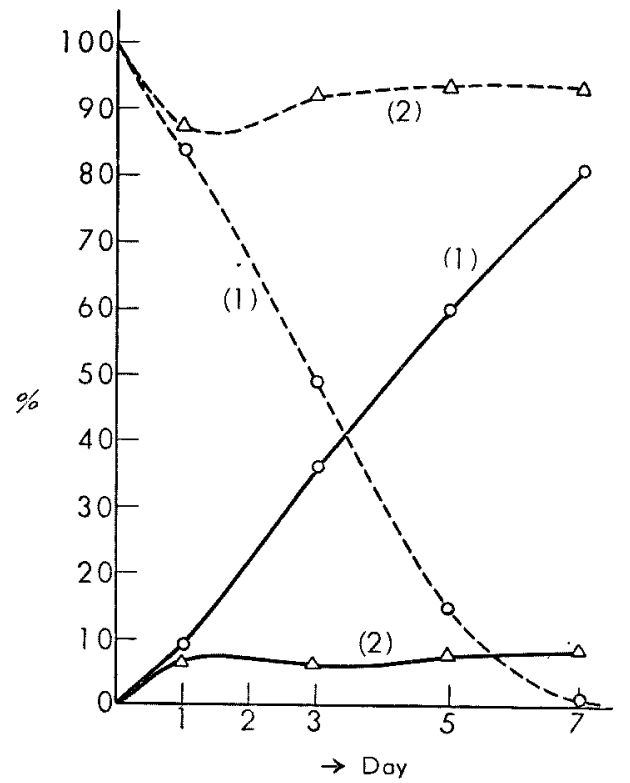

FIG. 2. Ammonia- and Amino-Nitrogen from DLAlanine (in complete medium).

(1), DL-alanine only; (2), DL-alanine +arsenite. Exprassions are the same as in Fig. 1.

already observed by $O \operatorname{tani}^{11}$ that glucose was equally available for this fungus as sucrose. At appropriate intervals, ammonia- and amino-nitrogen, remaining glucose and the dry weight of mycelium were estimated. The decrease of the amount of detectable aminonitrogen was expressed in per cent as alanine consumed. The decrease of the amount of alanine due to deamination was also involved in the data, but the formation of ammonia was far smaller throughout the present iucubation, that is, within the range of $0.69 \%$ to $2.52 \%$. The results are presented in Table $\mathrm{I}$.

Separation and Identification of Keto AcidsTwo mycelial felts were incubated in $40 \mathrm{ml}$ of the medium which consisted of $50 \mathrm{mg}$ of DL-norleucine, $20 \mathrm{ml}$ of $\mathrm{M} / 15$ phosphate buffer ( $\mathrm{pH} 6.9$ ), $5 \mathrm{ml}$ of M/20 potassium arsenite and $15 \mathrm{ml}$ of distilled water. Two hundred and fourty-five millilitre of a four-day old culture filtrate was concentrated under diminished pressure at $\mathrm{pH} 8.0 \sim 8.2$ and extracted with ether for three hours, followed by acidic ether extraction for five hours. The aqueous solution of the residual oily substance from the latter ether-extract was added with a small amount of $2 \mathrm{~N}$ hydrochloric acid and recon-

11) Y. Otani, Ann. Phytopatbolog. Sor. Japan, 17, 119, (1953). centrated under $14 \mathrm{~mm} \mathrm{Hg}$ at about $45^{\circ}$ The treatment of the distillate with 2,4-dinitrophenylhydrazine gave a yellow crystal mass which melted at about $130^{\circ}$. Recrystallization with acidic dilute ethanol resulted in a. long yellow needle of m.p. $148 \sim 9^{\circ}$ (Found: $\mathrm{C}, 46.31$; $\mathrm{H}, 4.38$. Calcd. for $\mathrm{C}_{12} \mathrm{H}_{14} \mathrm{O}_{6} \mathrm{~N}_{4}: \mathrm{C}, 46.45 ; \mathrm{H}, 4.51$.). In paper chromatography using butanol- $3 \%$ ammonia solvent, only one spot of $R_{F}=0.81 \sim 0.83$ was observed. This melting point is in good accordance with that of 2,4-dinitrophenylhydrazone of $\boldsymbol{\alpha}$-keto-caproic acid as

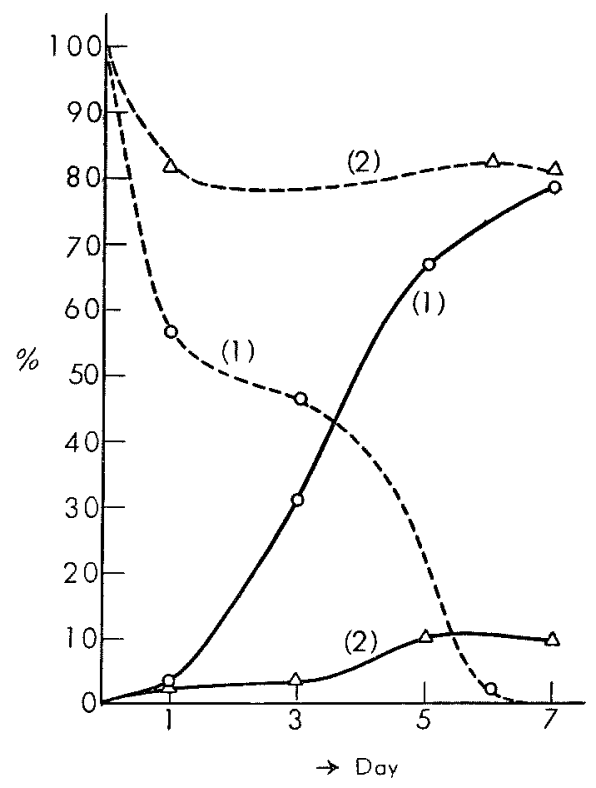

FIG. 3. Ammonia- and Amino-Nitrogen from DL- $\mathrm{Eg}$ Alanine (in phosphate buffer medium, $\mathrm{pH} 6.9$ ).

Expressions are the same as in Fig. 2.

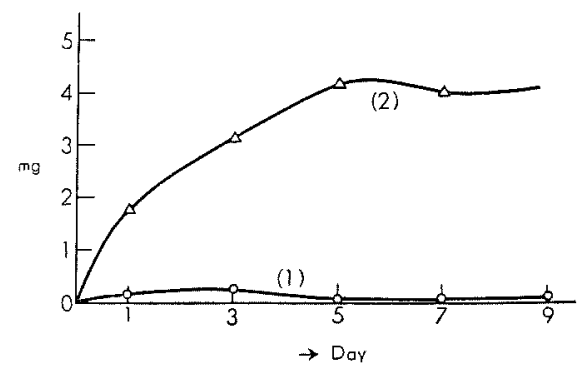

FIG. 4. Accumulation of Pyruvic Acid from DLAlanine.

Values express the amount of pyruvic acid in $60 \mathrm{ml}$ of phosphate buffer medium. (1), indicates the incubation added with no arsenite, and (2), added with arsenite. 


\section{Table I. Utilization of Alanine and Glucose}

\begin{tabular}{lcccccccccc}
\multicolumn{1}{c}{ Day } & 1 & 3 & 5 & 7 & 10 & 12 & 14 & 17 & 20 & 22 \\
Alanine consumed (\%) & 3.69 & 13.46 & 18.77 & 27.09 & 24.53 & 26.15 & 28.57 & 34.52 & 33.06 & 45.74 \\
Glucose consumed (\%) & 3.02 & 5.01 & 5.01 & 8.61 & 7.37 & 17.46 & 21.87 & 26.50 & 44.09 & 82.36 \\
Dry weight of mycelium (mg) & - & 5.0 & 11.9 & 32.1 & 48.6 & 84.0 & 83.6 & 98.0 & 137.4 & 174.6
\end{tabular}

Present medium contained $150 \mathrm{mg}$ of DLalanine and $792.5 \mathrm{mg}$ of D.glucose per flask. Figures express the average value of two flasks.

described by Krebs ${ }^{12)}$. Yield, Ca. $2.5 \mathrm{mg}$. From the residual solution remaining in the distillation flask a small amount of the same hydrazone was recovered. The same 2,4-dinitrophenylhydrazone was also obtained from the medium without arsenite, although the separation procedure was a little more difficult and its yield was unsatisfactory, compared to the case in which arsenite was added as just mentioned.

The separation and identification of pyruvic acid derivable from alanine was far easier, compared to the separation experiments conducted so far with other several amino acids. The condition of incubation was the same as in the case of norleucine. An amount of $110 \mathrm{ml}$ of the filtrate on the fifth day of incubation, which was strongly positive for Rothera's test, was treated in the same way as just described above. The 2,4-dinitrophenylhydrazone prepared from the acidic ether extract melted at $216 \sim 7^{\circ}$, on being recrystallized from ethanol solution and gave no depression in mixed melting point determination with an authentic sample. Yield, Ca. $7 \mathrm{mg}$. In paper chromatography (butanol$3 \%$ ammonia solvent; room temperature; ascending method) only one spot of $R_{F}=0.28 \sim 0.30$, i.e., anti-type of this hydrazone ${ }^{132}$ was observed. The separation of this keto acid from the medium without arsenite resulted in fail, as it was expected from the negligible Rothera's reaction of the filtrate. This fact is only characteristic of alanine among the ten amino acids used, that is, L-leucine, DL-valine, L-aspartic acid, Lphenylalanine, DL-methionine, glycine, DL-norleucine, DL-isoleucine, DL-alanine and DL- $\alpha$-aminobutyric acid, as a supplement in this series.

\section{DISCUSSION}

The formation of either $\alpha$-keto acid from leucine ${ }^{1)}$ and isoleucines) by Piricularia oryzae has already been established by the authors. This led us to attempt to confirm such metabolic conversion with the remaining six carbon amino acid, that is, norleucine and

12) H. A. Krebs, Z. Pbysiol. Chem., 217, 191, (1933).

13) T. Moriwaki, M. Katsuki and S. Tanaka, J. Chem. Sor. Japan. 76, 1367, (1955). thus, the formation of $\alpha$-ketocaproic acid from this amino acid was successfully recognized both in the presence and absence of arsenite. In the absence of arsenite this amino acid was easily deaminated (Fig. 1) as was observed with other natural amino acids and, in addition, norleucine was found to be utilized in the form of free amino acid itself, that is, $20 \sim 23$ per cent of the amino acid added up to the second day of incubation and 40 47 per cent up to the seventh day. This experimental result is somewhat different from the cases conducted with leucine ${ }^{1)}$ and valine ${ }^{3)}$, in which only a slight amount of each amino acid was utilized, not via its conversion to corresponding keto acid. In spite of uncertain natural occurrence of norleucine, the nutritional significance for some microorganisms has been examined by several investigators: growth-promoting effect for hiochi-bacteria ${ }^{14)}$, brewer's yeast ${ }^{15)}$, and Neurospora mutant ${ }^{16}$ ) and, to the contrary, inhibitory effect for $E$. coli17,18). In addition, its role in transamination $19,20,21$ ) and in oxidation by amino acid oxidase 22) was also observed. The present work seems to support further evidence that this amino acid is as much available as other natural amino acids, at least for some microorganisms. The amino nitrogen in the culture filtrate added with arsenite, however, was observed to increase again after the third

14) S. Teramoto and W. Hashida, J. Fermeatation Technol. Japan, 31, 334, (1953).

15) C. Rainbow, Cbem. Abstr., 47, 2261, (1953)

16) R. W. Bartatt and Wm. Ogata, $A m$. J. Botany, 41, 763. (1954).

17) D. Rowley, Nature, 171, 80, (1953)

18) W. Kellner and Hanna Hellmuth, Chem. Abstr., 49, 13355 (1955).

19) J. H. Wiame and R. Storck, Chem. Abstr., 47, 5487, (1953).

20) K. Heyns and Wolfgang Koch, Z. Pbysiol. Cbem., 288, 272, (1951).

21) L. Gehring, Cbem. Abstr., 49, 16061, (1955).

22) Y. Ptani, Ann. Phytopatbolog. Sor. Japan, 18, 159 (1954). 
day, in spite of the almost constant amount of ammonia produced. A similar tendency was observed in the case of $\alpha$-aminobutyric acids) and rarely in the case of alanine as a supplement. This may be due to the production of endogenous amino acids, but at present no any data explaining thoroughly such observation are available.

The nutritional availability of alanine as a nitrogen source for blast fungus was shown by Tomizawa7), and Otani22) has reported that amino acid oxidase secreted by present fungus is able to attack alanine. The present investigation was undertaken to examine the metabolic significance as a carbon source and thus the conversion of this amino acid to its $\alpha$-keto analogue. Sufficient utilization of alanine is proved from Fig. 2, and the same utilization process was also found in the phosphate buffer medium (Fig. 3). From this result it is established that it is possible to carry out such an experiment just mentioned, in the phosphate buffer, if necessary. The absorption and utilization in the form of alanine itself was 14.8 per cent and 25.4 per cent on the third and the fifth day, respectively, but in the presence of arsenite such utilization process was almost negligible, although seven to eight per cent ammonia was produced up to the third day. It is noteworthy that the accumulation of pyruvic acid was detected only in the presence of arsenite and that in the absence of this inhibitor approximate one-fortieth of the amount in the former case was estimated (Fig. 4). Similar results were obtained in the investigation of the aerobic degradation of alanine and glucose by $E$. coli and Clost. Perfringens ${ }^{23,24)}$ and of the metabolic behavior of Asp. niger growing in glucose media ${ }^{25)}$. These facts may probably be based upon the inhibitory effect of arsenite for the deami-

\footnotetext{
23) M. Kunimi and S. Hirano, Modicins and Bislogy Japan, 31, 197. (1954).

24) S. Hirano, Medicine and Biology Japan, 32, 262. (1954).

25) M. I. D. Chughtai, A. A. Parce and T. K. Walker, Biocbem. J., 47, 155, (1950).
}

nation of alanine and further metabolic process of pyruvic acid such as decarboxylation. The curve indicating the amount of pyruvic acid in the medium added with arsenite showed a similar tendency with that of ammonia produced (Fig. 2, Fig. 3), although the amount of pyruvic acid detected was somewhat lower than that calculated on the basis of the amount of ammonia liberated, which will show the possibility of utilization of some parts of pyruvic acid formed even in the presence of $5 \mathrm{ml}$ of $\mathrm{m} / 20$ arsenite. The other characteristic of alanine is that it is preferentially utilized by the present fungus, especially in a relatively earlier-stage of growth, to proper carbon source scuh as glucose (Table I), as it was recognized with glutamic acid by Tanaka9). The consumption of alanine was observed to be far superior to that of glucose during the first ten days of growth, thereafter, followed by a gradual increase of the consumption of glucose, though it did not reach the consumption of alanine. After the twentieth day of incubation, a remarkable increase of consumption of glucose, compared to the relatively slower increase of consumption of alanine, was recognized. This characteristic behavior of $P$. oryzae towards both gultamic acid and alanine may present one hopeful step towards the investigation of the biochemical relationship existing between this fungus and the host plant.

\section{SUMMARY}

(1) Piricularia oryzae is able to utilize DLnorleucine through its conversion to $\alpha$-ketocaproic acid, although, the natural occurrence of this amino acid still remains questionable. This keto acid is identified as its 2,4-dinitrophenylhydrazone.

(2) In the utilization of DL-alanine by $P$. oryzae some parts of this amino acid were utilized in the form of amino acid itself and other parts via prior conversion to pyruvic acid, but the accumulation of the present 
keto acid was observed only in the presence of arsenite, as was already recognized with other several microorganisms.

(3) It was established that alanine is preferentially utilized to a proper carbon source such as glucose, as it was recognized' with glutamic acid. This may present a probable step towards solving the problem of biochemical mechanism of invasion of this fungus. and its further growth in the host plant.

[Bull. Agr. Chem. Soc. Japan, Vol. 22, No. 2, p. 108 116, 1958]

\title{
Biochemical Studies of Piricularia oryzae
}

\begin{abstract}
Part VI. Behavior towards DL- $\alpha$-Aminobutyric Acid and DL-Isoleucine: Formation of $\alpha$-keto- $\beta$-Methylvaleric Acid from either Amino Acid.
\end{abstract}

By Tokuji Shimomura and Yukihiko Nakamura

Department of Agricullural Chemistry, Faculty of Agriculture", Hokkaido University

Received November 13, 1957

\begin{abstract}
When DL- $\alpha$-aminobutyric acid was used as a supplement in successive studies on the behavior of Piricularia oryzae towards amino acids, a keto acid differing from the $\alpha$-keto analogue of this amino acid was isolated as its 2,4-dinitrophenylhydrazone upon which this hydrazone was found to be identical with the 2,4-dinitrophenylhydrazone of $\alpha$-keto- $\beta$-methylvaleric acid which was formed from DL-isoleucine in another experiment. This finding indicates the formation of a six carbon compound from four carbon amino acid. The probable role of $\alpha-$ aminobutyric acid as an intermediate in the biosynthetic pathway of isoleucine has been discussed by several investigators, mainly based on the biochemically deficient mutant strain method and more recently on the isotopic competition method. Our present data seem to demonstrate direct evidence supporting the biosynthetic route of isoleucine in which $\alpha$-aminobutyric acid or its $\alpha$-keto analogue is included as a precursor of isoleucine. In addition, the presence of a close metabolic relationship between $\alpha$-aminobutyric acid and isoleucine may be suggested from the fact that pyruvic acid and $\alpha$-ketoglutaric acid were recognized together in respective experiment in which either $\alpha$-aminobutyric acid or isoleucine was used as the supplement in the presence of arsenite.
\end{abstract}

In the successive study of the behavior of Piricularia oryzae towards amino acids, DL- $\alpha$ aminobutyric acid was employed as the amino acid to be investigated. In this experiment a keto acid differing from the $\alpha$-keto analogue of this amino acid was isolated as its 2,4dinitrophenylhydrazone from the culture medium, which was assumed to be the 2,4dinitrophenylhydrazone of a six carbon keto acid, especially of $\alpha$-keto analogue of isoleucine, from some experimental data1). In order to obtain the sample comparable with the 2,4-dinitrophenylhydrazone from $\alpha$-aminobutyric acid just mentioned, and also in order to find the behavior towards isoleucine, a. similar incubation experiment as previous?) 1) T. Snimomura and Y. Nakamura, This Bulletin, 19. 178, 2) T. Shimomura and $Y$. Naknmura, part $V$ of this series. 